\title{
Development, validation and clinical evaluation of a broad-range pan-filovirus RT-qPCR
}

\section{Jääskeläinen, Anne J.}

2019-05

Jääskeläinen , A J , Sironen , T , Diagne , C T , Diagne , M M , Faye , M , Faye , O , Faye , O, Hewson , R, Mölsä , M , Weidmanng, M W , Watson, R, Sall , A A \& Vapalahti , O 2019 , ' Development, validation and clinical evaluation of a broad-range pan-filovirus RT-qPCR ' , Journal of Clinical Virology , vol. 114 , pp. 26-31 . https://doi.org/10.1016/j.jcv.2019.03.010

http://hdl.handle.net/10138/315580

https://doi.org/10.1016/j.jcv.2019.03.010

publishedVersion

Downloaded from Helda, University of Helsinki institutional repository.

This is an electronic reprint of the original article.

This reprint may differ from the original in pagination and typographic detail.

Please cite the original version. 


\title{
Development, validation and clinical evaluation of a broad-range pan- filovirus RT-qPCR
}

\author{
Anne J. Jääskeläinen ${ }^{\mathrm{a}, *}$, Tarja Sironen ${ }^{\mathrm{b}, \mathrm{c}}$, Cheikh Tidiane Diagne ${ }^{\mathrm{d}}$, Moussa Moïse Diagne $^{\mathrm{d}}$, \\ Martin Faye $^{\mathrm{d}}$, Oumar Faye ${ }^{\mathrm{d}}$, Ousmane Faye ${ }^{\mathrm{d}}$, Roger Hewson ${ }^{\mathrm{e}}$, Markos Mölsä ${ }^{\mathrm{f}}$, \\ Manfred W. Weidmann ${ }^{\mathrm{g}}$, Robert Watson ${ }^{\mathrm{e}}$, Amadou Alpha Sall ${ }^{\mathrm{d}}$, Olli Vapalahti ${ }^{\mathrm{a}, \mathrm{b}, \mathrm{c}}$ \\ ${ }^{a}$ Helsinki University and Helsinki University Hospital (HUSLAB), Department of Virology, Finland \\ ${ }^{\mathrm{b}}$ University of Helsinki, Department of Virology, Helsinki, Finland \\ ${ }^{\mathrm{c}}$ Faculty of Veterinary Medicine, Department of Veterinary Biosciences, University of Helsinki, Finland \\ ${ }^{\mathrm{d}}$ Institut Pasteur de Dakar, Pôle de virologie, Dakar, Senegal \\ ${ }^{\mathrm{e}}$ National Infection Service, Public Health England, Porton Down, Salisbury, United Kingdom \\ ${ }^{\mathrm{f}}$ National Institute for Health and Welfare, Biothreat unit, Centre for Military Medicine, Helsinki, Finland Centres for Biothreat Preparedness and for Military Medicine, \\ Finnish Defence Forces, Finland \\ ${ }^{\mathrm{g}}$ University of Stirling, Institute of Aquaculture, Stirling, United Kingdom
}

\section{A R T I C L E I N F O}

\section{Keywords:}

Ebola

Marburg

Sudan

Bundibugyo

Pan-Filo

\begin{abstract}
A B S T R A C T
Background: During the five decades since their discovery, filoviruses of four species have caused human hemorrhagic fever outbreaks: Marburg (MARV) marburgvirus, and Zaire (EBOV), Sudan (SUDV) and Bundybugyo (BDBV) ebolaviruses. The largest, devastating EBOV epidemic in West Africa in 2014-16, has been followed by outbreaks of MARV in Uganda, 2017, and EBOV in Democratic Republic of Congo, 2018, emphasizing the need to develop preparedness to diagnose all filoviruses.

Objectives: The aim of this study was to optimize a new filovirus RT-qPCR to detect all filoviruses, define its limits of detection (LOD) and perform a field evaluation with outbreak samples.

Study design: A pan-filovirus RT-qPCR targeting the L gene was developed and evaluated within the EbolaMoDRAD (Ebola virus: modern approaches for developing bedside rapid diagnostics) project. Specificity and sensitivity were determined and the effect of inactivation and PCR reagents (liquid and lyophilized format) were tested.

Results: The LODs for the lyophilized pan-filovirus L-RT-qPCR assay were 9.4 copies per PCR reaction for EBOV, 9.9 for MARV, 1151 for SUDV, 65 for BDBV and 289 for Taï Forest virus. The test was set at the Pasteur Institute, Dakar, Senegal, and 83 Ebola patient samples, with viral load ranging from 5 to 5 million copies of EBOV per reaction, were screened. The results for the patient samples were in $100 \%$ concordance with the reference EBOVspecific assay.

Discussion: Overall, the assay showed good sensitivity and specificity, covered all filoviruses known to be human pathogens, performed well both in lyophilized and liquid-phase formats and with EBOV outbreak clinical samples.
\end{abstract}

\section{Background}

Members of marburgvirus and ebolavirus genera in the family Filoviridae cause highly contagious illnesses with high mortality rate. There are five established species of ebolavirus: Zaire (EBOV), Bundibugyo (BDBV), Sudan (SUDV), Taï Forest (TAFV) and Reston (RESTV) viruses. All five can cause human infections, the first three have caused Ebola virus disease (EVD) outbreaks, TAFV has been associated with only one human case, whereas RESTV has been associated only with asymptomatic human seroconversions. Members of the Marburgvirus genus, consisting of the Marburg marburgvirus (MARV) and the Ravn virus (RAVV), also cause severe hemorrhagic fever in humans [1]. The third genus, Cuevavirus, is represented by the species Lloviu cuevavirus (LLOV), which hasn't yet been associated with

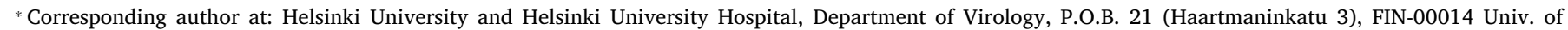
Helsinki, Finland.

E-mail address: anne.jaaskelainen@helsinki.fi (A.J. Jääskeläinen). 
human infections [2]. Filoviruses are thought to be zoonotic, and bats are considered the likely reservoirs of these viruses [3]. The Egyptian fruit bat, Rousettus aegypticus, has been identified as the host for MARV [4] while the very recently described Bombali ebolavirus (BOMV) and LLOV genome sequences were discovered in samples of insectivorous bats in Africa and Europe, respectively [2,5]. While the exact host of ebolaviruses has not been confirmed yet, genetic and serological evidence of ebolavirus infections have been detected in a few species of fruit bats and most recently insectivorous bats [5-9]. Advances in virus discovery techniques have also yielded detection of novel marburg- and cuevaviruses in bats in China [10,11] thus expanding the known range of filoviruses.

The largest Ebola outbreak to date took place in 2014-2016 in both rural and urban areas of Guinea, Sierra Leone and Liberia in West Africa. It was caused by the EBOV, with more than 28000 reported cases, including more than 11000 deaths (World Health Organization, WHO; http://www.who.int/csr/disease/ebola/en/). This epidemic highlighted the need for rapid detection of EBOV for disease containment. In response, novel diagnostic tools have been developed for rapid and safe identification of EBOV (reviewed in Clark et al. [12]). The development of efficacious ring vaccination and new treatment modalities further requires efficient diagnostics. Most recently, the WHO reported a new epidemic of EBOV in the Democratic Republic of the Congo (DRC) in May 2018, and an unrelated outbreak some $2500 \mathrm{~km}$ away in DRC in July 2018 calling for swift response yet again.

Between the EBOV outbreaks, a smaller outbreak of MARV occurred in Uganda in October 2017, emphasizing the need to develop preparedness to diagnose all filoviruses. Whereas many protocols have been developed recently for detection of EBOV, the detection methods covering the whole range of filoviruses still rely largely on the protocol by Panning et al. [13]. The discoveries of yet novel filoviruses [5] further underline the need to update and improve the preparedness for rapid and sensitive detection of filoviruses.

Nucleic acid testing is the gold standard for filovirus diagnostics due to high viral loads that become detectable in just a few days after infection [14]. Such diagnostic tools need to be set up both at the site of the epidemic, and at sites of potential importation. The EBOV outbreak in West Africa activated a broad laboratory response e.g. in Europe [15], with modern molecular diagnostics vastly available for detection of EBOV. For example, freeze-dried PCR reagents would ease the transport and use of the assays in harsh field conditions at the site of epidemic.

Critical steps in nucleic acid testing are biosafe sample preparation and transport. Different protocols for the inactivation of ebolaviruses have been investigated, such as Qiagen AVL buffer and the MagNa Pure Lysis buffer (MPLB; Roche Life Science, Espoo, Finland). The protocol for sampling directly into MPLB has been suggested [16] and this would enable biosafe transport, which is challenging nowadays. Two widely used inactivators are Triton ${ }^{\mathrm{TM}}$-X 100 , which interferes with lipid membranes, and lysis buffers containing guanidinium thiocyanate that lyse cells and inhibit nuclease activities. Neither of these can alone inactivate ebolaviruses, but parallel use of these two [e.g. Triton ${ }^{\mathrm{TM}}-\mathrm{X} 100$ together with AVL buffer from Viral RNA mini kit (Qiagen) or MPLB (Roche)] have been shown to fully inactivate ebolaviruses [16-18]. WHO has recommended the parallel usage of two different inactivation reagents.

\section{Objectives}

Here we aimed to develop and evaluate a broad-range pan-filovirus detection method allowing early identification of the causative agent of a filovirus outbreak. We also evaluated the freeze-dried and liquid formats, and possible effects that sample inactivation methods may have on the sensitivity of the assay. Finally, the method was put to test with a large panel of EVD outbreak patient samples.

\section{Study design}

\subsection{Pan-filo $L-R T-q P C R$}

Two different PCR reagents; Superscript ${ }^{\circ}$ III Platinum ${ }^{\circledast}$ One-step qRT-PCR System (Invitrogen, Carlsbad, CA, USA; later referred as the Invitrogen assay) and lyophilized one-step RT-qPCR reagents (Thermo Fisher Scientific, Massachusetts, USA; later referred as the Thermolyophilized assay) were used with the same primers, probes, and viral RNA panels.

A pan-filo L-RT-qPCR targeting the L gene was developed and evaluated. For the assay, two separate reactions were carried out for each sample in the same run.

In the first reaction, $160 \mathrm{nM}$ FAM-labelled Filo1 and Filo2 probes (adapted from Jääskeläinen et al. [19]; targeting ZEBOVs) in addition to ZEBOV/MARV reverse primers [480 nM of 5'-AATGCATCCAATTAA AAACATTC-3' [19], $240 \mathrm{nM}$ of 5'-AATGCATCCARTCRAATAAATTY-3'] and $240 \mathrm{nM}$ ZEBOV/MARV forward primers [5'-AACTGATTTAGAGAA ATACAATCTTGC-3' [19], 5'-CTGATCTTGAGAAATACAACCTCGC-3', 5'-ACTGATYTAGAGAAATACAAYCTYGC-3'], and $160 \mathrm{nM}$ VIC-labelled Filo3 and Filo4 probes [VIC- TTT ACA CGR CAT TTC ATA GAC TMGBNFQ and VIC- ACT GTA ATC GAT GTT ATG GT-MGBNFQ; mainly targeting MARVs] were used with final concentration of $2 \mathrm{mM} \mathrm{MgSO}_{4}$.

In the second reaction, a final concentration of $240 \mathrm{nM}$ of BDBV-RV primer [5'-AATGCATCCAATTGAATAAATTT-3'], 240 nM SUDV-RV [5'CATCCAATCAAAGACATTGC-3'], 320 nM FILO-FW [5'-ACMGACCTRGARAAATAYAACYTGGC-3'] in addition to 160 nM FAM-labelled Filo5probe [FAM- ATG AGT TTA CAG CTC CAT T-MGBNFQ; mainly BDBVs] and $160 \mathrm{nM}$ VIC-labelled Filo6 probe [VIC- TCA TCA AAT ATT GCA ACC AA-MGBNFQ; mainly targeting SUDVs] were used.

The Invitrogen assay in the Mx3005P qPCR System (Agilent Technologies Finland Oy, Espoo, Finland) was initially used to optimize the concentrations of the primers and probes. Both Invitrogen and Thermo-lyophilized assays were further tested by screening of different filoviruses, and to verify the specificity, and to test the PCR reagent ability to tolerate the inhibition effect of Triton ${ }^{\mathrm{TM}}-\mathrm{X} 100$ (Sigma-Aldrich, Espoo, Finland), MPLB (Roche), and AVL lysis buffer (Qiagen, Hilden, Germany). Finally, the limits of detection (LODs) were determined for the Thermo-lyophilized assay.

The RT-qPCR running protocol for Invitrogen-liquid assay was adapted from the EBOV assay described in Jääskeläinen et al. [19] and $7 \mu \mathrm{l}$ of template was used, and the protocol for Thermo-lyophilized assay $3.6 \mu \mathrm{l}$ of template was used and the run was carried out as follows: reverse transcription for $10 \mathrm{~min}$ at $45^{\circ} \mathrm{C}$, PCR initial activation step for $10 \mathrm{~min}$ at $95^{\circ} \mathrm{C}$ followed by 50 cycles of denaturation for $15 \mathrm{~s}$ at $95^{\circ} \mathrm{C}$, and annealing and extension for $70 \mathrm{~s}$ at $60^{\circ} \mathrm{C}$.

\subsection{Viral controls}

Quantified in vitro RNAs were produced using constructs for EBOV and MARV L genes (described in Jääskeläinen et al. [19]), in addition to synthetized L-gene constructs of SUDV and BDBV ebolaviruses (GeneArt $^{\mathrm{TM}}$ Plasmid Construction Service, Thermo Fischer Scientific). RNAs from inactivated whole virus controls of EBOV/Guinea C05 and EBOV/ Mayinga, MARV/Angola, RAVV, SUDV/Boniface, BDBV/E76, TAFV/ Ivory Coast and RESTV were obtained from Public Health England (PHE, Porton Down, Salisbury, UK). These viruses were cultivated in Vero E6 cells, inactivated, and RNA extracted using QIAamp Viral RNA Mini kit (Qiagen, Hilden, Germany) according to manufacturer's instruction.

\subsection{Specificity and inhibition tests}

For assessing specificity, EDTA-blood samples from 45 individuals that were sent for routine human herpesvirus 6 nucleic acid testing (later referred as HU-samples) in Helsinki University Hospital 
Table 1

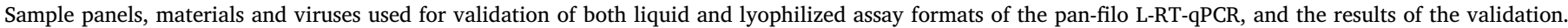

\begin{tabular}{|c|c|c|c|c|c|c|c|}
\hline \multirow[t]{3}{*}{ Panel/sample material } & \multirow[t]{3}{*}{ Microbial agent } & \multicolumn{2}{|c|}{$\begin{array}{l}\text { No of samples/tests, } \\
\text { neg/pos }\end{array}$} & \multicolumn{4}{|c|}{$\begin{array}{l}\text { Pan-filo L-RT-qPCR assays: Results from Liquid and Lyophilized platforms } \\
\text { compared }\end{array}$} \\
\hline & & \multirow[t]{2}{*}{$\begin{array}{l}\text { neg for } \\
\text { FILO RNA }\end{array}$} & \multirow[t]{2}{*}{$\begin{array}{l}\text { pos for } \\
\text { FILO RNA }\end{array}$} & \multicolumn{2}{|l|}{$\begin{array}{l}\text { neg for } \\
\text { FILO RNA }\end{array}$} & \multicolumn{2}{|l|}{$\begin{array}{l}\text { pos for } \\
\text { FILO RNA }\end{array}$} \\
\hline & & & & Liq (Invitrogen) & Lyo (Thermo) & Liq (Invitrogen) & Lyo (Thermo) \\
\hline NEG: & Negative sample panel & 45 & - & $45 / 45$ & $45 / 45$ & $0 / 45$ & $0 / 45$ \\
\hline Whole blood $^{\mathrm{a}}$ & Total & 45 & - & $45 / 45$ & $45 / 45$ & $0 / 45$ & $0 / 45$ \\
\hline NEG: & YFV (strains 17D) & 3 & - & $3 / 3$ & $3 / 3$ & $0 / 3$ & $0 / 3$ \\
\hline \multirow[t]{8}{*}{ Viruses $^{\mathrm{b}}$} & DENV1 (RKI) & 3 & - & $3 / 3$ & $3 / 3$ & $0 / 3$ & $0 / 3$ \\
\hline & DENV2 (RKI) & 3 & - & $3 / 3$ & $3 / 3$ & $0 / 3$ & $0 / 3$ \\
\hline & DENV3 (RKI) & 3 & - & $3 / 3$ & $3 / 3$ & $0 / 3$ & $0 / 3$ \\
\hline & DENV4 (RKI) & 3 & - & $3 / 3$ & $3 / 3$ & $0 / 3$ & $0 / 3$ \\
\hline & $\begin{array}{l}\text { LASV (strain Liberia, } \\
\text { RKI) }\end{array}$ & 3 & - & $3 / 3$ & $3 / 3$ & $0 / 3$ & $0 / 3$ \\
\hline & RVFV (strain RKI, RKI) & 3 & - & $3 / 3$ & $3 / 3$ & $0 / 3$ & $0 / 3$ \\
\hline & CCHFV (strain Hoti, RKI) & 3 & - & $3 / 3$ & $3 / 3$ & $0 / 3$ & $0 / 3$ \\
\hline & Total & 24 & - & $24 / 24$ & $24 / 24$ & $0 / 24$ & $0 / 24$ \\
\hline All FILO negatives & & \multicolumn{6}{|c|}{ 69/69 (100\%; 95CI 94.8-100\%) } \\
\hline \multirow[t]{10}{*}{ POS: Viruses/Viral RNAs } & $\begin{array}{l}\text { ZEBOV (strain Mayinga, } \\
\text { PHE) }\end{array}$ & - & 3 & $0 / 3$ & $0 / 3$ & $3 / 3$ & $3 / 3$ \\
\hline & MARV (strain Angola) & - & 2 & $0 / 2$ & $0 / 2$ & $2 / 2$ & $2 / 2$ \\
\hline & MARV (strain Ravn) & - & 2 & $0 / 2$ & $0 / 2$ & $2 / 2$ & $2 / 2$ \\
\hline & SUDV strain Boniface & - & 2 & $0 / 2$ & $0 / 2$ & $2 / 2$ & $2 / 2$ \\
\hline & BDBV strain E76 & - & 2 & $0 / 2$ & $0 / 2$ & $2 / 2$ & $2 / 2$ \\
\hline & $\begin{array}{l}\text { Taï Forest virus strain } \\
\text { Ivory Coast }\end{array}$ & - & 2 & $0 / 2$ & $0 / 2$ & $2 / 2$ & $2 / 2$ \\
\hline & Reston virus & - & 2 & $0 / 2$ & $0 / 2$ & $2 / 2$ & $2 / 2$ \\
\hline & MARV L-gene RNA (HU) & - & 15 & $0 / 15$ & $0 / 15$ & $15 / 15$ & $15 / 15$ \\
\hline & $\begin{array}{l}\text { ZEBOV L-gene construct } \\
\text { RNA (HU) }\end{array}$ & - & 15 & $0 / 15$ & $0 / 15$ & $15 / 15$ & $15 / 15$ \\
\hline & Total & - & 45 & $0 / 45$ & $0 / 45$ & $45 / 45$ & $45 / 45$ \\
\hline \multirow{16}{*}{$\begin{array}{l}\text { POS: } \\
38 \text { viral controls tested using RealStar } \\
\text { Filovirus RT-PCR } \mathrm{Kit}^{\mathrm{c}} \text { and Invitrogen } \\
\text { assay }\end{array}$} & Virus & \multicolumn{4}{|c|}{ Reference test: RealStar ${ }^{\circ}$ Filovirus } & \multicolumn{2}{|l|}{ Liq, Invitrogen } \\
\hline & & \multicolumn{4}{|c|}{5 parallel rxns, mean $\mathrm{Ct}( \pm \mathrm{SD})$} & \multicolumn{2}{|c|}{5 parallel rxns, mean Ct $( \pm S D)$} \\
\hline & $\begin{array}{l}\text { ZEBOV (strain Guinea, } \\
\text { PHE); } 100 \text { copies/rxn }\end{array}$ & \multicolumn{4}{|c|}{$5 / 5$, Ct $30.3( \pm 0.31)$} & \multicolumn{2}{|c|}{$5 / 5$, Ct $35.6( \pm 0.66)$} \\
\hline & 50 copies $/ \mathrm{rxn}$ & \multicolumn{4}{|c|}{$5 / 5$, Ct $32.3,( \pm 2.7)$} & \multicolumn{2}{|c|}{$4 / 5$, Ct $36.2( \pm 0.9)$} \\
\hline & 25 copies/rxn & \multicolumn{4}{|c|}{$1 / 5$, Ct $34.3(\mathrm{ND})$} & \multicolumn{2}{|c|}{$1 / 5$, Ct 36.1 (ND) } \\
\hline & Total ZEBOV & \multicolumn{4}{|c|}{$11 / 15$} & \multicolumn{2}{|l|}{$10 / 15$} \\
\hline & $\begin{array}{l}\text { SUDV L-gene (GeneArt), } \\
1500 \text { copies/rxn }\end{array}$ & \multicolumn{4}{|c|}{$5 / 5$, Ct $31.3( \pm 3.5)$} & $5 / 5$, Ct $38.3( \pm$ & \\
\hline & 150 copies/rxn & 2/5, Ct 33 . & $( \pm 3.8)$ & & & $1 / 5$, Ct $38.8(\mathrm{ND}$ & \\
\hline & 15 copies/rxn & $0 / 5$ & & & & $0 / 5$ & \\
\hline & Total SUDV & $7 / 15$ & & & & $6 / 15$ & \\
\hline & BDBV L-gene (GeneArt) ${ }^{\mathrm{d}}$, & 1250: Ct 3 & $4(\mathrm{ND})$ & & & 1250: Ct $33.4(\mathrm{~N}$ & \\
\hline & $1250 ; 625 ; 125$ copies/ & 625: Ct 32 . & (ND); & & & 625: Ct 36.5 (ND & \\
\hline & rxn & 125:Ct 35. & (ND) & & & 125: Ct 36.3 (ND & \\
\hline & 62.5 copies $/ \mathrm{rxn}$ & $1 / 5$, Ct 30 & ND) & & & $4 / 5$, Ct $38.1( \pm$ & \\
\hline & Total BDBV & $4 / 8$ & & & & $7 / 8$ & \\
\hline & Altogether & $22 / 38$ & & & & $23 / 38$ & \\
\hline
\end{tabular}

POS, positive; NEG, negative; FILO, filoviruses; rxn, reaction; ND, not determined; ZEBOV, Zaire ebolavirus; MARV, Marburg virus; BDBV, Bundibugyo virus; SUDV, Sudan ebolavirus; HU, University of Helsinki; PHE, Public Health England; RKI, Robert Koch Institute; DENV, denguevirus; LASV, Lassa virus; YFV, yellow fever virus; CCHFV, Crimean-Congo Hemorrhagic virus; RVFV, Rift-Valley Fever virus.

${ }^{a}$ EDTA-Blood samples (Helsinki University Hospital, HUSLAB, Finland).

b RNA extractions from inactivated virus cultivations kindly provided by Prof. Niedrig (Robert Koch Institute, Germany).

c RealStar" Filovirus Screen RT-PCR Kit (altona Diagnostics GmbH, Hamburg, Germany). Only liquid phase Invitrogen pan-filo L RT-qPCR assay was carried out parallel with RealStar ${ }^{\circ}$ Filovirus Screen RT-PCR Kit.

${ }^{\mathrm{d}}$ Only one reaction was carried out.

(HUSLAB, Helsinki, Finland; anonymous samples, research permit TYH2017257), were extracted using MagNa Pure 96 automated system and nucleic acid kits (MPLB lysis; Roche Life Science, Espoo, Finland), and tested using the Invitrogen and Thermo-lyophilized assays. In addition, for testing other viral hemorrhagic fever agents, the RNAs from inactivated whole virus controls of Lassa virus (strain Liberia; LASV), Dengueviruses 1-4 (DENV1-4), Yellow fever virus (strain 17D; YFV), Rift Valley fever virus (strain RKI; RVFV) and Crimean-Congo hemorrhagic virus (strain Hoti; CCHFV) were extracted using QIAamp Viral RNA Mini kit (Qiagen) (Table 1).

For Triton ${ }^{\mathrm{TM}}-\mathrm{X} 100$ (Sigma-Aldrich, Espoo, Finland) inhibition tests, excess amount of Triton $^{\mathrm{TM}}$-X 100 was added to EDTA-blood samples in final volume of $10 \%$ in order to test any PCR inhibition effect due to the reagent in both Invitrogen and Thermo-lyophilized assays. Tritontreated (10 $\mathrm{min}$ at room temperature) EDTA-blood samples were extracted using AVL and QIAamp Viral RNA Mini kit (Qiagen). In addition, EDTA-blood samples without extra Triton treatment were extracted using MPLB and MagNA Pure extraction system (Roche). Different amounts of EBOV, MARV, SUDV or BDBV RNAs were spiked in the extracted samples, and both Thermo-lyophilized and Invitrogen assays were carried out (Fig. 1). 


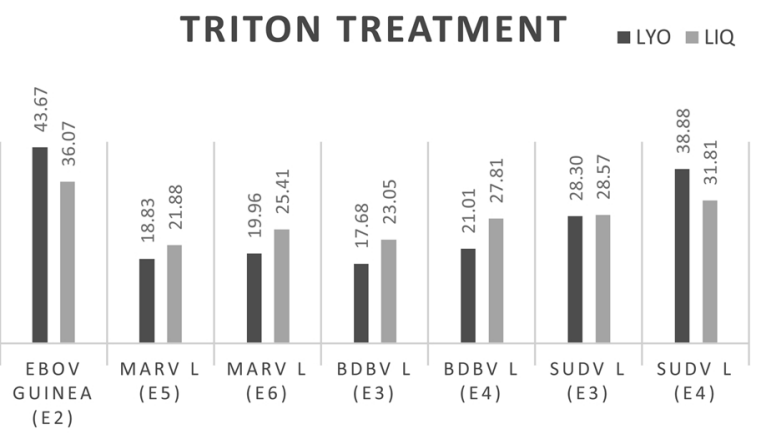

b

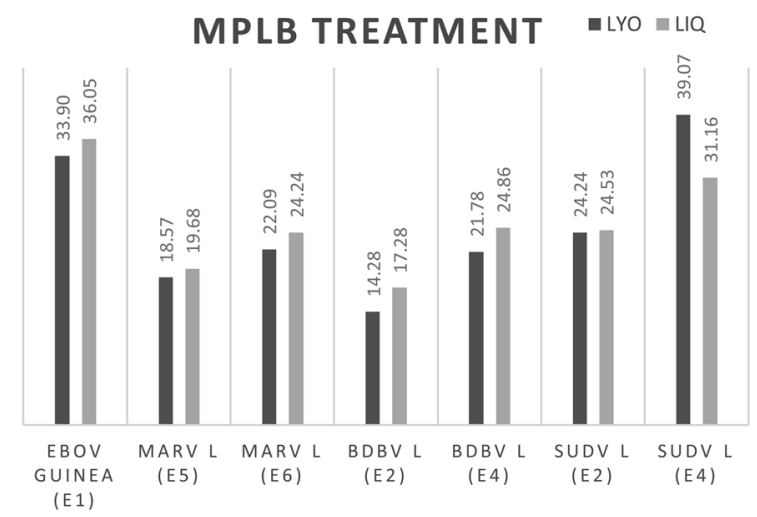

Fig. 1. (a) and (b) Comparing the performance of the pan-filo L-RT-qPCR using Thermo-lyophilized and Invitrogen-liquid assays for potential inhibitory effects of different reagents and inactivation methods [Fig. 1a.,Triton ${ }^{\mathrm{TM}}-\mathrm{X} 100$ treatment (Triton) or Fig. 1b. Magna Pure Lysis buffer (MPLB)].

$\mathrm{Ex}, 10^{-\mathrm{x}}\left(\mathrm{E} 1=10^{-1}, \mathrm{E} 2=10^{-2} \ldots\right)$ dilution, single replicate; MARV L, L-gene construct of Marburg virus; ZEBOV Guinea, RNA extract of Zaire ebolavirus; BDBV L, L-gene construct of Bundibugyo virus; SUDV L, L-gene construct of Sudan ebolavirus; negat, negative.

Triton: EDTA-blood Triton ${ }^{\mathrm{TM}}$-X 100 treated and extracted using Qiagen kit, spiked with filoviral RNA.

MPLB: EDTA-blood, Magna Pure LC extracted, using Magna Pure Lysis buffer, spiked with filoviral RNA.

Over Ct 40 results are not considered real positive until confirmed by another test if used in diagnosis of filoviral disease in clinical settings. Here Ct-values are listed due to the comparison purposes.

\subsection{Sensitivity}

Sensitivity of the pan-filo L-RT-qPCR was tested using the Thermolyophilized assay and serial dilutions of quantified RNA transcripts (Qubit, Thermo Fisher Scientific) of EBOV, MARV, SUDV and BDBV Lgene, and TAFV (PHE, quantified). Five parallel reactions and Probit Regression (SPSS, IBM) were used to determine LODs. The Invitrogen assay was tested in parallel with the WHO-approved RealStar ${ }^{\oplus}$ Filovirus RT-PCR Kit (Altona Diagnostics GmbH, Hamburg, Germany) using ZEBOV strain Guinea (range 5-1000 genome copies/PCR reaction; PHE), SUDV L-gene RNA (range 7-1.5E6 genome copies/PCR reaction) and BDBV L-gene RNA (range 13-1.25E6 genome copies/PCR reaction) (Table 1). In addition, whole virus controls (Table 1, PHE) were tested to screen different filovirus targets.

\subsection{Screening of ebola patient samples}

Samples were collected in Guinea between December 2014 and May
2015 as part of the Institute Pasteur de Dakar (IPD) diagnostics activities of suspected EVD cases (under an emergency response mandate from the government of Guinea and WHO, ref 0235/14/GUI/CPC; all patients agreed to be tested for Ebola virus infection and leftover samples to be used for further investigations). Suspect Ebola patients were defined as any person with recent or past sudden onset of fever and having been in contact with a suspected, probable or confirmed case of EVD, or any person with sudden onset of fever and at least three of the following symptoms: headaches, anorexia/loss of appetite, lethargy, myalgia, arthralgia, breathing difficulties, or any person with inexplicable bleeding. Eighty-three serum samples from acute cases were extracted using QIAamp Viral RNA Mini kit (Qiagen) according to manufacturer's instructions in IPD, Senegal. These were all EBOV nucleic acid positive using the reference EBOV NP-RT-qPCR (Weidmann et al. [20]; using modified forward primer of 5'-ATGATGGARGCTACGGCG-3' and probe 5'-CARAGTTACTCGGAAAACGGCAT) with viral loads ranging from 5 RNA copies to 5.5 million RNA copies per reaction. In IPD, the pan-filo L-RT-qPCR and EBOV NP-RT-qPCR were carried out in parallel using QuantiTect ${ }^{\oplus}$ Probe RT-PCR kit (Qiagen) and $5 \mu \mathrm{l}$ of template for performance comparison.

\section{Results}

Both Invitrogen and Thermo-lyophilized pan-filo L-RT-qPCR assays tested negative for HU-samples $(N=45)$, as well as for LASV, DENV1-4, YFV, RVFV and CCHFV, indicating analytical specificity of $100 \%$ (95CI: 94.8-100\%; Table 1). In addition, both assays detected EBOV/Guinea C05, EBOV/Mayinga, MARV/Angola, RAVV, SUDV/Boniface, BDBV/ E76, TAFV/Ivory Coast and RESTV (Table 1).

The LODs (SPSS, Probit, 95CI) for Thermo-lyophilized assay were 9.9 copies/PCR reaction for MARV, 9.4 for EBOV, 65 for BDBV, 1151 for SUDV and 289 for TAFV (PHE).

The Invitrogen pan-filo L-RT-qPCR assay was as good as the RealStar Filovirus Screen RT-PCR Kit for EBOV, however, for BDBV and SUDV samples there were minor differences (Table 1).

Triton $^{\mathrm{TM}}$-X 100 (Sigma-Aldrich), AVL (Qiagen) or MPLB (Roche) did not interfere the Invitrogen or Thermo-lyophilized one-step assays (Fig. 1).

All of the patient samples tested (83) from IPD (Senegal) were positive for EBOV nucleic acids using our pan-filo L-RT-qPCR assay and EBOV NP-RT-qPCR (Fig. 2) indicating 100\% analytical sensitivity. The LOD for EBOV NP-RT-qPCR was 4 copies/PCR reaction (tested in University of Helsinki; SPSS, Probit, 95CI).

\section{Discussion}

Filoviruses were first isolated more than 50 years ago, and outbreaks with high mortality have subsequently been caused by four viruses, MARV ( 9 times) SUDV (5 times), BDBV (twice) and EBOV (14 times) (WHO). For containing and restricting the epidemics, including establishing control measures and therapeutics, accessible, rapid and reliable diagnostic tests are essential. IgM and IgG detection assays are used to confirm resolved disease or to diagnostically monitor samples of cases beyond the diagnostic window for molecular detection. While rapid antigen detection tests with varying sensitivity and specificity have been developed for EBOV detection [21,22], nucleic acid detection remains the cornerstone of diagnostics. It is a challenge to find simple protocols, particularly primer and probe sequences applicable to all pathogenic filoviruses, yet tests covering all filoviruses would be essential in early identification of outbreaks as well as occasional cases in endemic regions or travelers. Adding such a test and its evaluation is what we report here. However, in future, the lyophilized protocol would benefit from lyophilized primers and probes in mastermixes making the protocol more suitable for field conditions.

After the West African EBOV epidemic, a variety of methods, inactivation protocols and handling procedures have been studied 


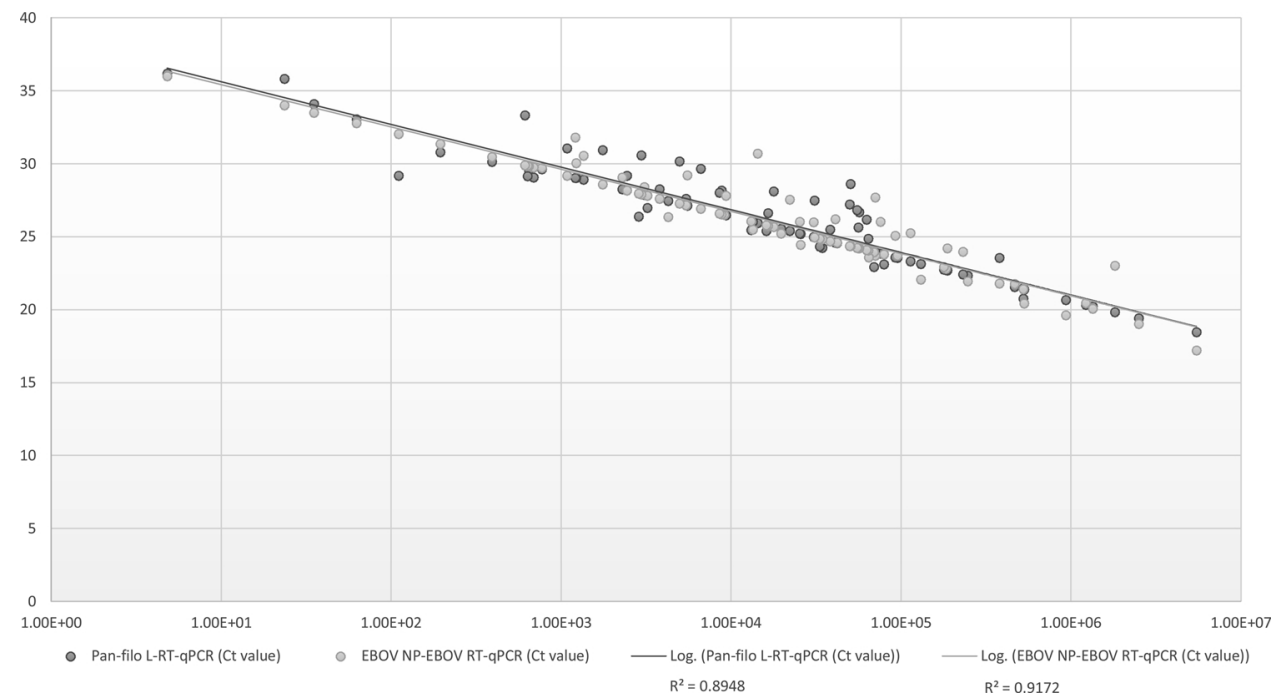

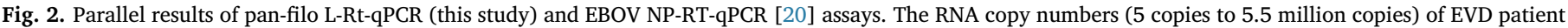
samples are presented in the $\mathrm{X}$-axis (logarithmic scale, $\log _{10}$ ) and Ct values in the Y-axis. $\mathrm{R}^{2}$, R-squared.

[16-18]. Most of the detection methods, however, have been based on the detection of only EBOV nucleic acids, and this limitation can cost time for diagnosis of other filoviruses. At the moment there is only one commercial pan-filovirus kit (RealStar ${ }^{\oplus}$ Filovirus Screen RT-PCR Kit) approved by WHO that also detects MARV, others still aim to detect EBOV antigen or nucleic acids (WHO; http://www.who.int/medicines/ ebola-treatment/emp_ebola_diagnostics/en/). These WHO-approved tests include Liferiver ${ }^{\mathrm{TM}}$ Ebola Virus (EBOV) Real Time RT-PCR kit (Shanghai ZJ BioTech Co., Ltd) which can be used for detection EBOV, SUDV, TAFV, BDBV, and Xpert Ebola Test (Cepheid AB, Sweden), FilmArray $^{\mathrm{TM}}$ Biothreat-E (BioFire Defence LLC, USA) which both only detect EBOV.

The 2-well pan-filo L-RT-qPCR assay described here detected all the tested strains of MARV, EBOV, BDBV, SUDV, TAFV, as well as RESTV. In addition, we were able to validate the pan-filo L-RT-qPCR assay with clinical samples from the West-African EBOV outbreak with excellent performance. Overall, the assay achieved better performance for EBOV and MARV than rest of the tested targets, but was still at the same level as WHO-approved RealStar Filovirus Screen RT-PCR kit. With this in mind, it's recommended to test several samples from patient suspected for VHF to avoid false negative results at the early onset of disease.

The specificity was $100 \%$, and the lyophilized or liquid assays were not affected by common chemical inactivation reagents, i.e. MPLB, AVL or Triton ${ }^{\mathrm{TM}}-\mathrm{X}$ 100. These results are in line with Rosenstierne et al. [16] who tested MPLB and AVL buffers.

Based on the sequences, the pan-filo L-RT-qPCR primers and probes are not a perfect match for BOMV (MF319185; forward primer 4, reverse 2, and VIC-probe 3 mismatches) or other bat-related filoviruses in GenBank (National Center for Biotechnology Information, USA). However, when screening Kenyan bat samples, with the pan-filo L-RTqPCR we detected one sample positive for filovirus. The bat-related filovirus was later sequenced and identified as BOMV [23].

We conclude that the developed assays, both lyophilized and liquid phase, can be used effectively to screen samples from patients suspected for any known filoviral hemorrhagic fever, and both marburg and ebolaviruses can be detected.

\section{Credit author statement}

Anne J. Jääskeläinen: Designing the pan-filo RT-qPCR method and primers and probes, writing and text editing, performing the PCRs in Finland.

Tarja Sironen: Performing PCRs in Finland, writing and text editing.
Cheikh Tidiane Diagne, Moussa Moïse Diagne, Martin Faye, Oumar Faye, Ousmane Faye: EBOV patients in Senegal, performing PCRs in Senegal, writing, text editing.

Amadou Alpha Sall: Supervision in Senegal, editing.

Markos Mölsä: Responsible of freeze-dried PCR reagents, text editing.

Roger Hewson, Manfred W Weidmann, Robert Watson: Supervision in UK, filoviral RNA. controls, designing Weidmann PCR method, writing and text editing, language editing.

Olli Vapalahti: Supervision in Finland, writing and text editing.

\section{Funding}

This project is part of the EbolaMoDRAD (Ebola virus: modern approaches for developing bedside rapid diagnostics) consortium, which has received funding from the Innovative Medicine Initiative 2 Joint Undertaking under grant agreement $\mathrm{N}^{\circ} 115843$. This Joint Undertaking receives support from the European Union's Horizon 2020 research and innovation programme and EFPIA. This work was also funded by Helsinki University Hospital (HUSLAB; research permit TYH2017257).

\section{Conflict of interest}

There are no conflicts of interest.

\section{Acknowledgements}

We thank the Robert Koch-Institut in Berlin, Germany, for providing the viral standard preparations (DENV, YFV, CCHFV, LASV) for diagnostic purposes. We thank HUSLAB PCR team personnel, Johanna Martikainen (University of Helsinki) and Minttu Kaloinen (University of Helsinki) for technical assistance.

\section{References}

[1] L. Nyakarahuka, J. Ojwang, A. Tumusiime, S. Balinandi, S. Whitmer, S. Kyazze, S. Kasozi, M. Wetaka, I. Makumbi, M. Dahlke, J. Borchert, J. Lutwama, U. Ströher, P.E. Rollin, S.T. Nichol, T.R. Shoemaker, Isolated case of Marburg virus disease, Kampala, Uganda, 2014, Emerg. Infect. Dis. 23 (2017) 1001-1004.

[2] A. Negredo, G. Palacios, S. Vázquez-Morón, F. González, H. Dopazo, F. Molero, J. Juste, J. Quetglas, N. Savji, M. de la Cruz Martínez, J.E. Herrera, M. Pizarro, S.K. Hutchison, J.E. Echevarría, W.I. Lipkin, A. Tenorio, Discovery of an ebolaviruslike filovirus in Europe, PLoS Pathog. 7 (2011) e1002304.

[3] K.J. Olival, D.T.S. Hayman, Filoviruses in bats: current knowledge and future directions, Viruses 6 (2014) 1759-1788. 
[4] J.S. Towner, B.R. Amman, T.K. Sealy, S.A. Carroll, J.A. Comer, A. Kemp, R. Swanepoel, C.D. Paddock, S. Balinandi, M.L. Khristova, P.B. Formenty, C.G. Albarino, D.M. Miller, Z.D. Reed, J.T. Kayiwa, J.N. Mills, D.L. Cannon, P.W. Greer, E. Byaruhanga, E.C. Farnon, P. Atimnedi, S. Okware, E. KatongoleMbidde, R. Downing, J.W. Tappero, S.R. Zaki, T.G. Ksiazek, S.T. Nichol, P.E. Rollin, Isolation of genetically diverse Marburg viruses from Egyptian fruit bats, PLoS Pathog. 5 (2009) e1000536.

[5] T. Goldstein, S.J. Anthony, A. Gbakima, B.H. Bird, J. Bangura, A. Tremeau-Bravard, M.N. Belaganahalli, H.L. Wells, J.K. Dhanota, E. Liang, M. Grodus, R.K. Jangra, V.A. DeJesus, G. Lasso, B.R. Smith, A. Jambai, B.O. Kamara, S. Kamara, W. Bangura, C. Monagin, S. Shapira, C.K. Johnson, K. Saylors, E.M. Rubin, K. Chandran, W.I. Lipkin, J.A.K. Mazet, The discovery of Bombali virus adds further support for bats as hosts of ebolaviruses, Nat. Microbiol. 3 (2018) 1084-1089 Erratum in: Nat. Microbiol. 2018, 3:1486.

[6] E.M. Leroy, B. Kumulungui, X. Pourrut, P. Rouquet, A. Hassanin, P. Yaba, A. Délicat, J.T. Paweska, J.P. Gonzalez, R. Swanepoel, Fruit bats as reservoirs of Ebola virus, Nature 38 (2005) 575-576.

[7] R. Biek, P.D. Walsh, E.M. Leroy, L.A. Real, Recent common ancestry of Ebola Zaire virus found in a bat reservoir, PLoS Pathog. 2 (2006) e90.

[8] R. Swanepoel, S.B. Smit, P.E. Rollin, P. Formenty, P.A. Leman, A. Kemp, F.J. Burt, A.A. Grobbelaar, J. Croft, D.G. Bausch, H. Zeller, H. Leirs, L.E. Braack, M.L. Libande, S. Zaki, S.T. Nichol, T.G. Ksiazek, J.T. Paweska, International scientific and technical committee for Marburg hemorrhagic fever control in the Democratic Republic of Congo. Studies of reservoir hosts for Marburg virus, Emerg. Infect. Dis. 13 (2007) 1847-1851.

[9] E.D. Laing, I.H. Mendenhall, M. Linster, D.H.W. Low, Y. Chen, L. Yan, S.L. Sterling, S. Borthwick, E.S. Neves, J.S.L. Lim, M. Skiles, B.P.Y. Lee, L.F. Wang, C.C. Broder, G.J.D. Smith, Serologic evidence of fruit bat exposure to filoviruses, Singapore, 2011-2016, Emerg. Infect. Dis. 24 (2018) 114-117.

[10] B. He, Y. Feng, H. Zhang, L. Xu, W. Yang, Y. Zhang, X. Li, C. Tu, Filovirus RNA in fruit bats, China, Emerg. Infect. Dis. 21 (2015) 1675-1677.

[11] X.L. Yang, Y.Z. Zhang, R.D. Jiang, H. Guo, W. Zhang, B. Li, N. Wang, L. Wang, C. Waruhiu, J.H. Zhou, S.Y. Li, P. Daszak, L.F. Wang, Z.L. Shi, Genetically diverse filoviruses in Rousettus and Eonycteris spp. Bats, China, 2009 and 2015, Emerg. Infect. Dis. 23 (2017) 482-486.

[12] D.J. Clark, J. Tyson, A.D. Sails, S. Krishna, H.M. Staines, The current landscape of nucleic acid tests for filovirus detection, J. Clin. Virol. 103 (2018) 27-36.

[13] M. Panning, T. Laue, S. Olschlager, M. Eickmann, S. Becker, S. Raith, M.C. Courbot, M. Nilsson, R. Gopal, A. Lundkvist, A. di Caro, D. Brown, H. Meyer, G. Lloyd, B.M. Kummerer, S. Gunther, C. Drosten, Diagnostic reverse-transcription polymerase chain reaction kit for filoviruses based on the strain collections of all
European biosafety level 4 laboratories, J. Infect. Dis. 196 (Suppl. 2) (2007) S199-204.

[14] A.C. Shurtleff, C.A. Whitehouse, M.D. Ward, L.H. Cazares, S. Bavari, Pre-symptomatic diagnosis and treatment of filovirus diseases, Front. Microbiol. 6 (2015) 108

[15] C.B. Reusken, R. Mögling, P.W. Smit, R. Grunow, G. Ippolito, A. Di Caro, M. Koopmans, Status, quality and specific needs of Ebola virus diagnostic capacity and capability in laboratories of the two European preparedness laboratory networks EMERGE and EVD-LabNet, Euro Surveill. 23 (19) (2018).

[16] M.W. Rosenstierne, H. Karlberg, K. Bragstad, G. Lindegren, M.L. Stoltz, C. Salata, A.M. Kran, S.G. Dudman, A. Mirazimi, A. Fomsgaard, Rapid bedside inactivation of Ebola virus for safe nucleic acid tests, J. Clin. Microbiol. 54 (2016) 2521-2529.

[17] J.E. Burton, L. Easterbrook, J. Pitman, D. Anderson, S. Roddy, D. Bailey, R. Vipond, C.B. Bruce, A.D. Roberts, The effect of a non-denaturing detergent and a guanidinium-based inactivation agent on the viability of Ebola virus in mock clinical serum samples, J. Virol. Methods 250 (2017) 34-40.

[18] J.J.A. van Kampen, A. Tintu, H. Russcher, P.L.A. Fraaij, Reusken CBEM, M. Rijken, J.J. van Hellemond, P.J.J. van Genderen, R. Koelewijn, M.D. de Jong, E. Haddock, R.J. Fischer, V.J. Munster, M.P.G. Koopmans, Ebola virus inactivation by detergents is annulled in serum, J. Infect. Dis. 216 (2017) 859-866.

[19] A.J. Jääskeläinen, K. Moilanen, K. Aaltonen, N. Putkuri, T. Sironen, H. KallioKokko, O. Vapalahti, Development and evaluation of a real-time EBOV-L-RT-qPCR for detection of Zaire ebolavirus, J. Clin. Virol. 67 (2015) 56-58.

[20] M. Weidmann, E. Mühlberger, F.T. Hufert, Rapid detection protocol for filoviruses, J. Clin. Virol. 30 (2004) 94-99.

[21] N.F. Walker, C.S. Brown, D. Youkee, P. Baker, N. Williams, A. Kalawa, K. Russell, A.F. Samba, N. Bentley, F. Koroma, M.B. King, B.E. Parker, M. Thompson, T. Boyles, B. Healey, B. Kargbo, D. Bash-Taqi, A.J. Simpson, A. Kamara, T.B. Kamara, M. Lado, O. Johnson, T. Brooks, Evaluation of a point-of-care blood test for identification of Ebola virus disease at Ebola holding units, Western Area, Sierra Leone, January to February 2015, Euro Surveill. 20 (12) (2015).

[22] M.J. Broadhurst, J.D. Kelly, A. Miller, A. Semper, D. Bailey, E. Groppelli, A. Simpson, T. Brooks, S. Hula, W. Nyoni, A.B. Sankoh, S. Kanu, A. Jalloh, Q. Ton, N. Sarchet, P. George, M.D. Perkins, B. Wonderly, M. Murray, N.R. Pollock, ReEBOV antigen rapid test kit for point-of-care and laboratory-based testing for Ebola virus disease: a field validation study, Lancet 386 (2015) 867-874.

[23] K.M. Forbes, P.W. Webala, A.J. Jääskeläinen, S. Abdurahman, J. Ogola, M.M. Masika, I. Kivistö, H. Alburkat, I. Plyusnin, L. Levanov, E.M. Korhonen, E. Huhtamo, D. Mwaengo, T. Smura, A. Mirazimi, O. Anzala, O. Olli Vapalahti, T. Sironen, Bombali ebolavirus is widespread in Africa: evidence from an infected Mops condylurus bat in south-east Kenya, Emerg. Infect. Dis. (2019) Proofs accepted, (in press). 